Research Article

\title{
Research on Load Characteristics of Axle-Box Bearing Raceway under Wheel-Rail Excitation
}

\author{
Xiaokang Liao $\mathbb{D}^{1},{ }^{1}$ Cai Yi $\mathbb{D},{ }^{2}$ Fengyu Ou, ${ }^{3}$ Yi Zhang, ${ }^{2}$ Zili Chen, ${ }^{4}$ and Jianhui Lin ${ }^{2}$ \\ ${ }^{1}$ School of Electrical Engineering, Southwest Jiaotong University, Chengdu 611756, Sichuan, China \\ ${ }^{2}$ State Key Laboratory of Traction Power, Southwest Jiaotong University, Chengdu 610031, Sichuan, China \\ ${ }^{3}$ School of Mechanical Engineering, Southwest Jiaotong University, Chengdu 610031, Sichuan, China \\ ${ }^{4}$ School of Mathematics, Southwest Jiaotong University, Chengdu 611756, Sichuan, China \\ Correspondence should be addressed to Cai Yi; yicai@swjtu.edu.cn
}

Received 23 August 2021; Revised 12 October 2021; Accepted 12 November 2021; Published 27 November 2021

Academic Editor: Xingxing Jiang

Copyright $\odot 2021$ Xiaokang Liao et al. This is an open access article distributed under the Creative Commons Attribution License, which permits unrestricted use, distribution, and reproduction in any medium, provided the original work is properly cited.

\begin{abstract}
With the increasing speed of high-speed trains, the service conditions of axle-box bearing system worsen, and meanwhile, the dynamic performance of the axle-box bearing directly affects the operational safety. However, the dynamic interactions of the axle-box bearing in the traditional vehicle-track system are often ignored. In this paper, a vehicle-track coupling dynamic model considering axle-box bearing has been built, and the effectiveness of the model is proved by field tests. Dynamic performance of the axle-box bearing has been analyzed and discussed through numerical simulations under different working conditions. Comparing the roller-raceway contact load characteristics under different working conditions, results show that the peak values of roller-outer raceway contact load with wheel-polygonal excitation are basically the same with those without wheel-rail excitation. However, most of the peak values of roller-outer raceway contact force under track irregularity and comprehensive excitation conditions are far greater than those under wheel-polygonal excitation and no wheel-rail excitation conditions, which indicates that the impact of track irregularity on the contact load is dominant.
\end{abstract}

\section{Introduction}

In recent years, the high-speed train in China has developed rapidly owing to its multiple advantages, such as security, comfort, high-speed, and eco-friendliness. With the increasing speed of high-speed trains, the service conditions of the axle-box bearing system worsen, leading to new challenges. The axle-box is a key component of high-speed trains, which is supported by double row tapered roller bearings. The double row tapered roller bearings have high strength against axial and radial loads. The dynamic performance of the axle-box bearing affects the operational security of high-speed trains directly. Even worse, an unexpected bearing failure may lead to train derailment, resulting in enormous economic loss and operating unsafety [1].

Some researchers have focused on the dynamic performance of tapered roller bearing without considering the coupling effects of the vehicle and the track. Palmgren [2],
Jones [3], and Harris [4] established a numerical model of rolling bearing, to analyse the mechanical relationship between the roller and inner/outer raceway of the rolling bearing. In order to understand the dynamic performance of tapered roller bearings, various investigations have been carried out that focused on determination of bearing characteristics. Andréason [5] analyzed the load distribution in a tapered roller bearing under the condition of radial and axial loads. Andréason's model was further improved by Liu [6] for investigating the influence of misalignment angles on the dynamic performance of a tapered roller bearing under combined loads. Nevertheless, both Liu and Andréason supposed that the displacements of tapered roller bearings are known, rather than setting them as unknown variable quantities. This factor obviously limits the applicability of the model. Tong and Hong [7] investigated the characteristics of the tapered roller bearing such as displacements, contact forces between roller and inner/outer raceway and flange, contact angle between roller and flange, load 
distribution along roller, and stiffness matrix, when the tapered roller bearing sustained combined radial and moment loads. He set the displacements of tapered roller bearings as unknown variables and determined them by iteratively solving the roller and bearing equilibrium equations. Recently, Houpert [8] improved the previous study to deduct an analytical approach to determining loads and moments of tapered roller bearings as a function of the given displacement with neglecting the influence of centrifugal and gyroscopic moment.

Previous investigations on the tapered roller bearings have focused on vehicle-track system dynamics. With the increasing speed of high-speed trains, the influence of wheel-rail excitation on the axle-box bearing becomes more and more complex, and meanwhile, the service conditions of axle-box bearing system worsen. Garg and Dukkipati [9] and Wickens [10] originated the classical vehicle dynamics theory, which focused only on the dynamic performance of a vehicle system. Zhai et al. [11, 12] developed a vehicle-track coupled dynamics model to investigate the dynamic performance of vehicle subsystems and track subsystems, and the model was validated experimentally. The similar concept has been adopted in many studies of railway dynamics [13-15]. Recently, Wang et al. $[16,17]$ developed a new vehicle-track coupled dynamics model that considered the axle-box bearing and studied the coupling effects on the vibration characteristics of the tapered roller bearings. However, the friction between the internal components of bearing is ignored, and all bearing rollers have the same angular motion. Zha et al. $[18,19]$ established a double row tapered roller bearing model with 6 degrees of freedom roller and 6 degrees of freedom cage based on the vehicle-track coupling dynamics model, investigated the vibration characteristics of axlebox bearing, and studied the contact load characteristics of roller raceway under track excitation. However, the wheelpolygonal excitation and comprehensive wheel-rail excitation are not considered, which cannot truly reflect the interaction between roller and raceway of bearing when vehicle is running.

Previous studies on vehicle systems have usually not considered the effects of bearings, and the studies of bearings have not considered the coupling effects between the bearings and the connected components of the vehicle. The main objective of this paper is to construct a vehicletrack-bearing coupled dynamics model to investigate the motion and interaction between the components of the axle-box bearings. When the axle-box bearings sustain external loads exerted by bogie and those generated within wheel-rail contact, the forces of the roller-inner/outer raceway will change complexly. Considering the motion and interaction between the components of the axle-box bearings, the loads of the roller-inner/outer raceway are calculated. The statistical characteristics are used to analyse the motion of internal components of the axle-box bearing under track irregularity excitation, wheelsetpolygon excitation, and comprehensive excitation. The research results have certain guiding significance for revealing the motion law of components under track excitation and evaluating the bearing life of high-speed train.

\section{Dynamic Model and Experimental Verification}

2.1. Vehicle-Track Coupled Dynamic Model. The vehicle is composed of a car body, two bogie frames, four wheelsets, and eight axle-boxes. The car body is supported on two bogies through the secondary suspension at either end, and the bogie frames are joined to the wheelset through the primary suspension system. The primary and secondary suspensions are modeled as equivalent springs and viscous dampers along three translational directions. The details of the vehicle-track coupled dynamics model [20] are shown in Figures 1 and 2.

Based on the constructed vehicle-track coupled dynamics model, the vehicle-track system is numerically simulated. The main simulation parameters of the vehicle subsystem are given in Table 1.

2.2. Experimental Verification. In order to verify the effectiveness of the constructed vehicle-track model, the field tests are carried out in the China's high-speed railway network. The installation position of the accelerometer on the axle-box is shown in Figure 3. Limited by the test conditions at that time, the tested bogie was equipped with the 19th order polygonal wheel, and we measured the lateral vibration acceleration of the axle-box near the polygonal wheel at the speed of $245 \mathrm{~km} / \mathrm{h}$. The speed and wheelpolygon order of the built vehicle-track model are consistent with those in the field tests.

Figure 4 shows the lateral acceleration of the axle-box in time and frequency domain. It can be seen that the amplitude of the axle-box lateral vibration acceleration in simulation and experiment is close in time domain, which is within the allowable error range of engineering calculation. The dominant frequency of $447.5 \mathrm{~Hz}$ is obviously seen in the simulation and experiment frequency domain, which is consistent with the fault frequency of the 19th order polygonal wheel. The experiment not only verifies the correctness of the vehicle model but also proves the effectiveness of the vehicle model under the wheel-polygonal excitation. Therefore, the vehicle-track coupling dynamic model built in this paper can well describe the physical characteristics of the vehicle-track system.

2.3. Axle-Box Bearing Model. Axle-box bearing usually consists of an inner raceway, an outer raceway, several rollers, and a cage, and the motion and interaction between the components of the axle-box bearing are complex [21]. In this paper, a model considering the contact between the roller and the guiding flange of the inner raceway is developed, and the bearing force diagram is shown in Figure 5.where $Q_{e}$ and $Q_{i}$ represent the contact force between the roller and outer/inner raceway, respectively. $Q_{f}$ indicates the contact force between the outer end of the roller and guiding flange of the inner raceway, and $\alpha_{e}, \alpha_{i}$, 


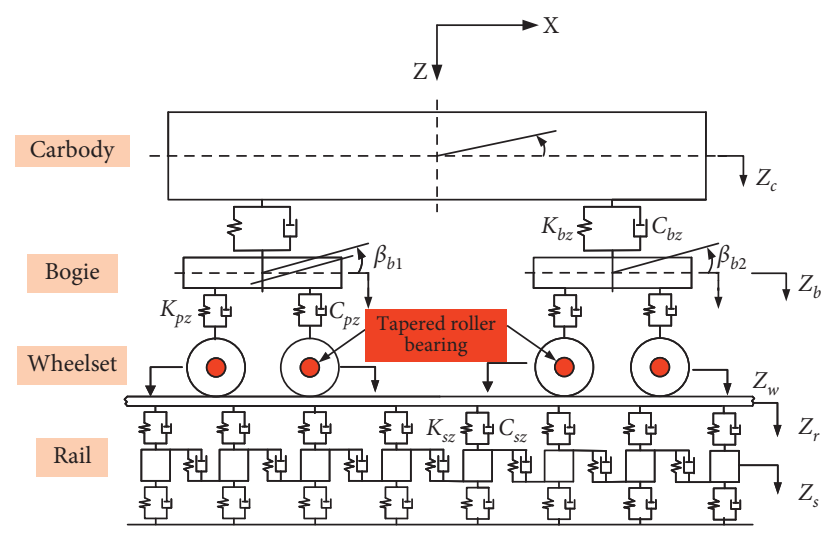

FIgURE 1: Vertical vehicle-track coupled dynamics model.

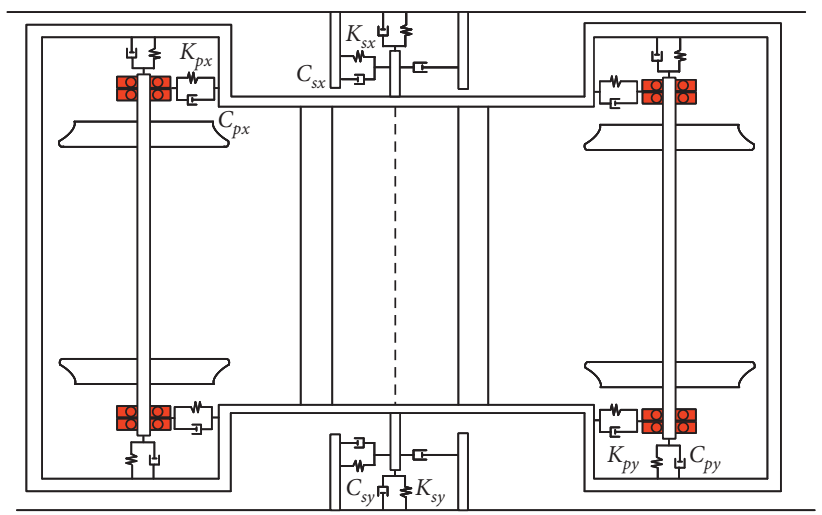

FIGURE 2: Lateral vehicle-track coupled dynamics model.

TABLE 1: Main parameters of vehicle model.

\begin{tabular}{lcc}
\hline Specification & Value & Unit \\
\hline Car body mass & 33786 & $\mathrm{~kg}$ \\
Bogie frame mass & 2056 & 1627 \\
Wheelset mass & $109500 / 1655300 / 1562300$ & $\mathrm{~kg}$ \\
Mass moment of car body about $x / y / z$ axes & $1390 / 2590 / 3800$ & $\mathrm{~kg}$ \\
Mass moment of bogie frame about $x / y / z$ axes & $825 / 132 / 830$ & $\mathrm{~kg} \cdot \mathrm{m}^{2}$ \\
Mass moment of wheelset about $x / y / z$ axes & 0.92 & $\mathrm{~kg} \cdot \mathrm{m}^{2}$ \\
Wheel rolling circle diameter & $919800 / 919800 / 886500$ & $\mathrm{~m}$ \\
Stiffness of primary suspension (longitudinal/lateral/vertical) & 10000 & $\mathrm{~N} / \mathrm{m}$ \\
Damping of primary suspension (vertical) & $12800 / 128000 / 225000$ & $\mathrm{~N} \cdot \mathrm{m} / \mathrm{s}$ \\
Stiffness of secondary suspension (longitudinal/lateral/vertical) & $15000 / 10000$ & $\mathrm{~N} / \mathrm{m}$ \\
Damping of secondary suspension (lateral/vertical) & $\mathrm{N} \cdot \mathrm{m} / \mathrm{s}$ \\
\hline
\end{tabular}

and $\alpha_{f}$ are the contact angle of the roller-outer raceway, roller-inner raceway, and roller-guiding flange, respectively.

$$
\left\{\begin{array}{l}
Q_{e} \sin \alpha_{e}-Q_{i} \sin \alpha_{i}-Q_{f} \sin \alpha_{f}=0 \\
Q_{e} \sin \alpha_{e}-Q_{i} \sin \alpha_{i}+Q_{f} \sin \alpha_{f}-F_{c}=0
\end{array}\right.
$$

The centrifugal force of rollers can be determined by

$$
F_{c}=\frac{1}{2} m_{r} D_{p} w_{c}^{2}
$$

where $m_{r}$ is mass of the roller, $D_{p}$ is the pitch diameter of double-row tapered roller bearing, and $w_{c}$ is the orbital angular velocity of the roller, and it can be formulated by

$$
w_{c}=\frac{w_{i}}{2 D_{p}}\left(D_{p}-D_{b} \cos \frac{\alpha_{i}+\alpha_{e}}{2}\right),
$$

where $w_{i}$ is the rotating speed of inner raceway and $D_{b}$ is the average diameter of the tapered roller.

When the tapered roller bearing is running, the rotation frequency of the inner raceway can be expressed as 


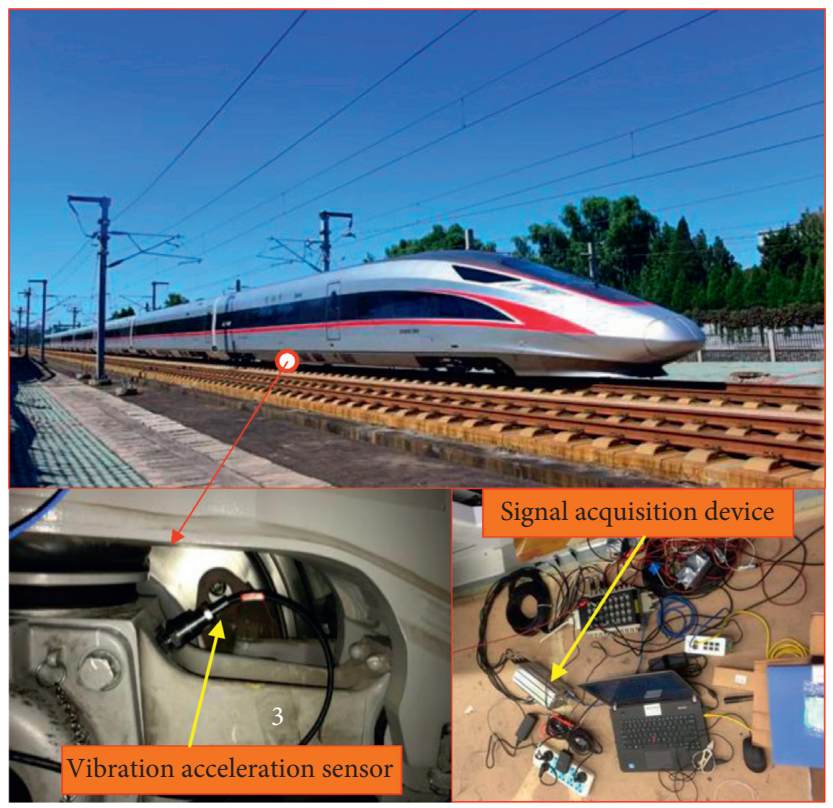

FIGURE 3: Installation of acceleration sensor at the test site.

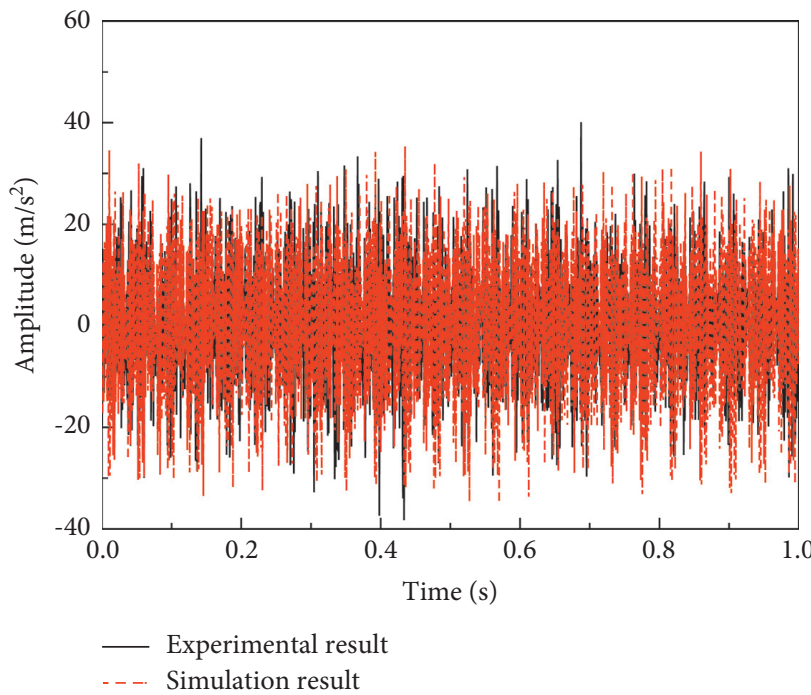

(a)

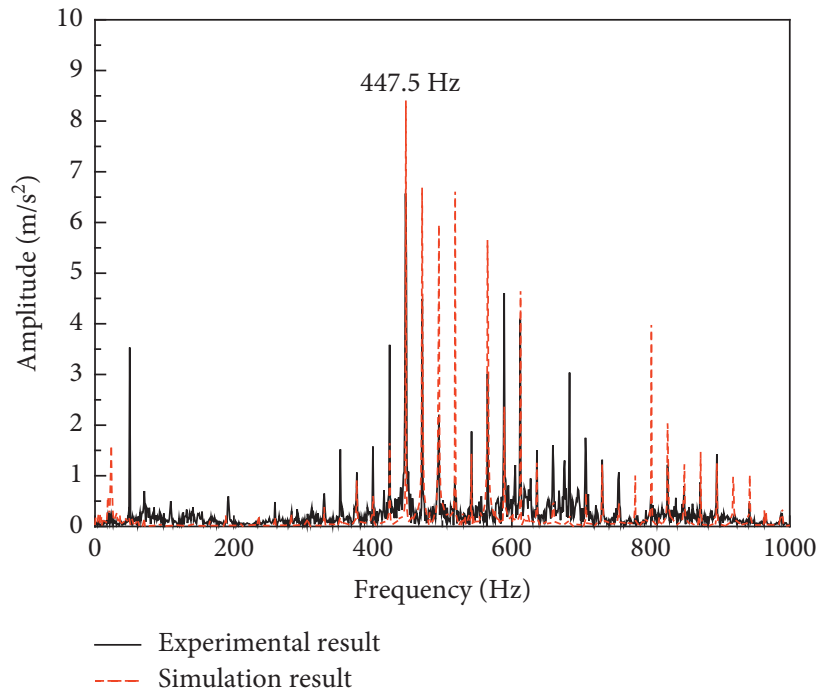

(b)

Figure 4: Validation of the vehicle-track model by comparison with experimental test: lateral acceleration of the axle-box in time domain (a) and frequency domain (b).

$$
f_{i}=\frac{v}{2 \pi R}
$$

where $v$ is the speed of the train (unit: $\mathrm{m} / \mathrm{s}$ ) and $R$ is nominal rolling radius of the wheel. The cage rotation frequency can be given by the following formula:

$$
f_{c}=\frac{f_{i}}{2}\left(1-\frac{D_{b}}{D_{p}} \cos \alpha_{i}\right) .
$$

The empirical formula proposed by Palmgren [2] is usually used to calculate the relationship between the elastic convergence and the contact load in the contact of the tapered roller bearing.

$$
\delta=3.81\left[\frac{1-v_{1}^{2}}{\pi E_{1}}+\frac{1-v_{2}^{2}}{\pi E_{2}}\right]^{0.9} \frac{Q^{0.9}}{l^{0.8}},
$$

where $E_{1}$ is elastic modulus of roller, $E_{2}$ is elastic modulus of the inner/outer raceway respectively, $v_{1}$ is Poisson's ratio of roller, and $v_{2}$ is Poisson's ratio of the inner/outer raceway, respectively. $Q$ is the contact load, and $l$ is effective length of the roller. And then, the total contact force $Q_{\text {mje }}$ between the roller and outer raceway can be obtained by 


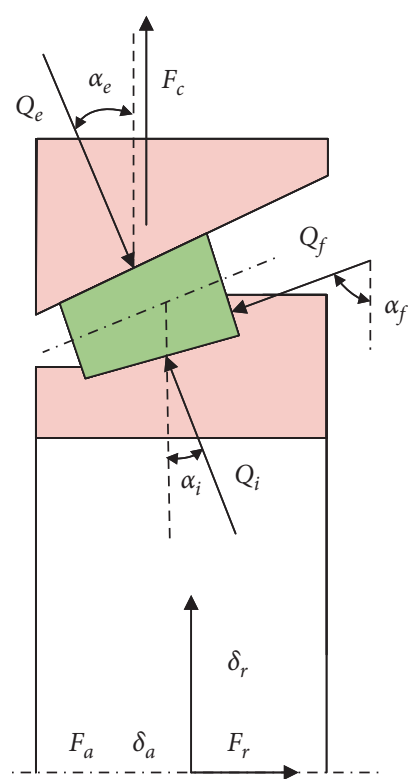

FIGURE 5: Schematic diagram of bearing force.

$$
\left\{\begin{array}{l}
Q_{m j e}=K_{m} \delta_{m}^{10 / 9}, \\
K_{m}=6.24 \times 10^{4} \times l^{0.84} D_{b}^{10 / 9}\left[1+c_{i}^{0.9} \cos \left(\alpha_{e}-\alpha_{i}\right)\right],
\end{array}\right.
$$

where $K_{m}$ is total contact stiffness coefficient between the roller and inner/outer raceway and $c_{i}$ is an index, which can be obtained by

$$
c_{i}=\frac{\sin \left(\alpha_{e}-\alpha_{i}\right)}{\sin \left(\alpha_{i}+\alpha_{f}\right)} .
$$

As presented in Figure 6, when the bearing has radial and axial displacements, considering the initial clearance $\left(C_{r}\right)$ in the radial direction, the total displacement of each roller along the contact normal direction of the outer raceway can be expressed as

$$
\left\{\begin{array}{l}
\delta_{1 j n}=\left[\delta_{r} \cos \varphi_{1 j}-0.5 C_{r}\left(1-\cos \varphi_{1 j}\right)\right] \cos \alpha_{e}+\delta_{a} \sin \alpha_{e}, \\
\delta_{2 j n}=\left[\delta_{r} \cos \varphi_{2 j}-0.5 C_{r}\left(1-\cos \varphi_{2 j}\right)\right] \cos \alpha_{e}-\delta_{a} \sin \alpha_{e}
\end{array}\right.
$$

where $\delta_{1 j n}$ and $\delta_{2 j n}$ are the total compression along the normal direction of the outer raceway contact line and $\delta_{r}$ and $\delta_{a}$ are the radial and axial displacements of the inner raceway relative to the outer raceway.

Based on the analysis above, the bearing mechanical balance equation can be expressed as

$$
\left\{F_{r}-\sum_{i=1}^{2} \sum_{j=-8}^{8} Q_{m j e} \cos \alpha_{e} \cos \varphi_{i j}=0, F_{a}-\sum_{i=1}^{2} \sum_{j=-8}^{8} Q_{m j e} \sin \alpha_{e}=0 .\right.
$$

Presuming that the vehicle is running at a constant speed on a straight line under the condition of no wheel-rail excitation and the bearing only bears the radial load generated by the gravity of the vehicle body parts, regardless of the initial radial clearance $C_{r}$, the normal displacement between the roller and the outer raceway at each position can be expressed as

$$
\delta_{m j e}=\delta_{r} \cos \varphi_{i j} \cos \alpha_{e} .
$$

Presuming that the maximum contact load between No. 0 roller and the outer raceway is $Q_{\max }$, the mathematical relationship between the roller-outer raceway contact load in the loaded zone and $Q_{\max }$ can be expressed as

$$
Q_{m j e}=Q_{\max } \cos \varphi_{i j}^{10 / 9} \text {. }
$$

According to the bearing mechanical balance equation (10), the theoretical contact load distribution model under the condition that the bearing only bears radial load and no radial clearance is presented as follows:

$$
F_{r}-Q_{\max } \cos \alpha_{e} \sum_{i=1}^{2} \sum_{j=-8}^{8} \cos \varphi_{i j}^{19 / 9}=0 .
$$

According to the equation above, when the relative displacement of the axle-box and wheelset at the bearing seat is known, the force acting on the inner raceway and outer raceway can be calculated. The axle-box bearing parameters of a high-speed train are shown in Table 2.

When the tapered roller bearing is running, the circumferential direction can be divided into two zones: the loaded zone and the unloaded zone. The roller in the loaded zone not only bears the centrifugal force and gravity along the circumference but also bears all the load of the spindle. The roller in the unloaded zone only bears centrifugal force and gravity. The outer raceway is divided into zones as shown in Figure 7, and the top of the raceway is defined as zone zero. On the roller, numbers in the clockwise direction are positive, and those in the counterclockwise direction are negative.

\section{Wheel-Rail Excitation Model}

3.1. Wheel-Polygonal Model. According to whether there is a dominant polygon of a certain order, the polygon wheel can be divided into periodic irregularity and nonperiodic irregularity. The polygonal wheel wear is full circle noncircular wear, which is characterized by the periodic change of wheel radius along the circumference of the wheel surface. To research the influence of polygonal wheel wear upon the 


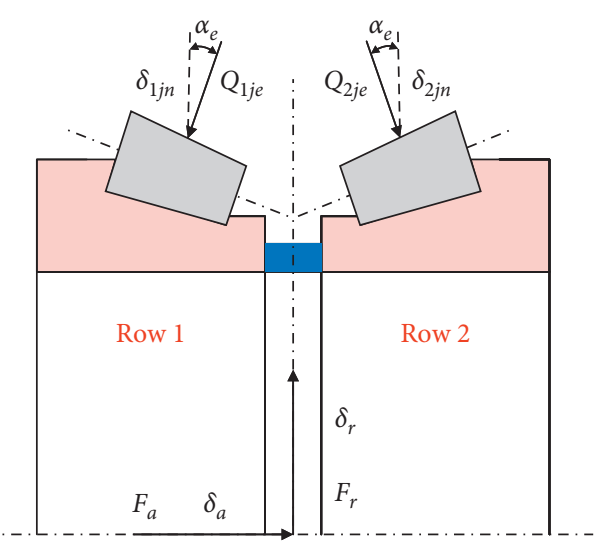

Figure 6: Double-row tapered roller bearing.

TABLE 2: Structure parameters of axle-box bearing.

\begin{tabular}{lc}
\hline Structure parameters & Value \\
\hline Diameter of outer raceway $(\mathrm{mm})$ & 240 \\
Width of outer raceway $(\mathrm{mm})$ & 160 \\
Diameter of inner raceway $(\mathrm{mm})$ & 130 \\
Average diameter of roller $(\mathrm{mm})$ & 26.5 \\
Number of rollers in each row & 17 \\
Contact angle of roller-outer raceway & $11^{\circ} 40^{\prime}$ \\
Contact angle of roller-inner raceway & $10^{\circ}$ \\
\hline
\end{tabular}

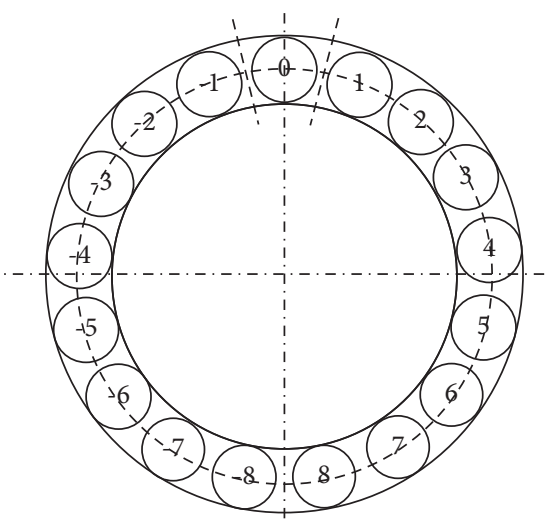

FIgURE 7: Outer raceway zone division.

axle-box bearings, the harmonic function is used to simulate the change in this radius [22]:

$$
\left\{\begin{array}{l}
\Delta r=A \sin \left(N \beta+\beta_{0}\right), \\
r(\beta)=R-\Delta r
\end{array}\right.
$$

where $A$ is the magnitude of polygonal wear, $N$ is the order of the polygonal wheel, $\beta$ is the angle of wheel rotation, $\beta_{0}$ is the phase angle, and $\Delta r$ is the wheel diameter difference along its circumference.

3.2. Track Irregularity Model. Through the calculation of the vehicle-track coupling contact relationship from Figures 1 and 2, it can be obtained that the relative displacement of the left and right wheel of the $n$th wheelset is

$$
\left\{\begin{array}{l}
\delta Z_{L n}=Z_{w n}(t)-\left(\Delta Z_{\text {Lwnt }}-\Delta Z_{L w n 0}\right) \\
\delta Z_{R n}=Z_{w n}(t)-\left(\Delta Z_{\text {Rwnt }}-\Delta Z_{\text {Rwn } 0}\right),
\end{array}\right.
$$

where $Z_{w n}(t)$ is the vertical displacement of the centroid of the $n$th wheelset, $\Delta Z_{\mathrm{Lwnt}}$ is the minimum vertical distances from the wheel to the rail at the left side, $\Delta Z_{\mathrm{R} w n t}$ is the minimum vertical distances from the wheel to the rail at the right side, $\Delta Z_{\mathrm{L} w n 0}$ is the left minimum vertical distances at initial moment, and $\Delta Z_{\mathrm{R} w n 0}$ is the right minimum vertical distances at initial moment.

According to the Hertz nonlinear contact theory, the wheel-rail vertical force can be expressed as

$$
p(t)=\left[\frac{1}{G} \delta Z(t)\right]^{3 / 2},
$$

where $G$ is a constant related to the Hertzian wheel-rail contact and $\delta Z(t)$ is the elastic deformation of the wheel-rail contact in the normal direction.

In the calculation, in both models, the train is running along a straight line at the speed of $250 \mathrm{~km} / \mathrm{h}$. The measured Beijing-Tianjin high-speed track spectrum is used as the track random irregularity excitation [16], as illustrated in Figure 8 .

\section{Load Characteristics of the Axle-Box Bearing Raceway}

4.1. Contact Load Characteristics of the Roller-Raceway without Wheel-Rail Excitation. The simulation calculation is carried out for the axle-box bearing under the condition of no wheel-rail excitation, and the contact load history between the rollers of row 1 /row 2 and the outer raceway at the same position is obtained, as shown in Figure 9. The contact load distribution curve between the rollers of row 1 and the inner/outer raceway is shown in Figure 10.

Under the condition of no wheel-rail excitation, the bearing runs smoothly, the roller-outer raceway contact load on both sides is consistent basically, and the load changes periodically with the vehicle running. The maximum rollerouter raceway contact load is $7.26 \mathrm{kN}$, which appears on the row 1 of the tapered roller bearing.

As can be seen from Figure 10, compared with the theoretical load distribution, the actual contact load enters the loaded zone ahead of time. In the unloaded zone, there is no contact between the roller and the inner raceway, but there is a small contact collision between the roller and the outer raceway under the influence of gravity and centrifugal force. In the loaded zone, the contact load between the roller and the inner/outer raceway is basically equal due to the small centrifugal force of the roller.

The contact load spectrum calculated by the fast Fourier transform is shown in Figure 11. The amplitude of 1 4 times cage rotation frequency is the largest, which is dominant in the frequency spectrum and reflects the variation law of the loaded zone and unloaded zone. 

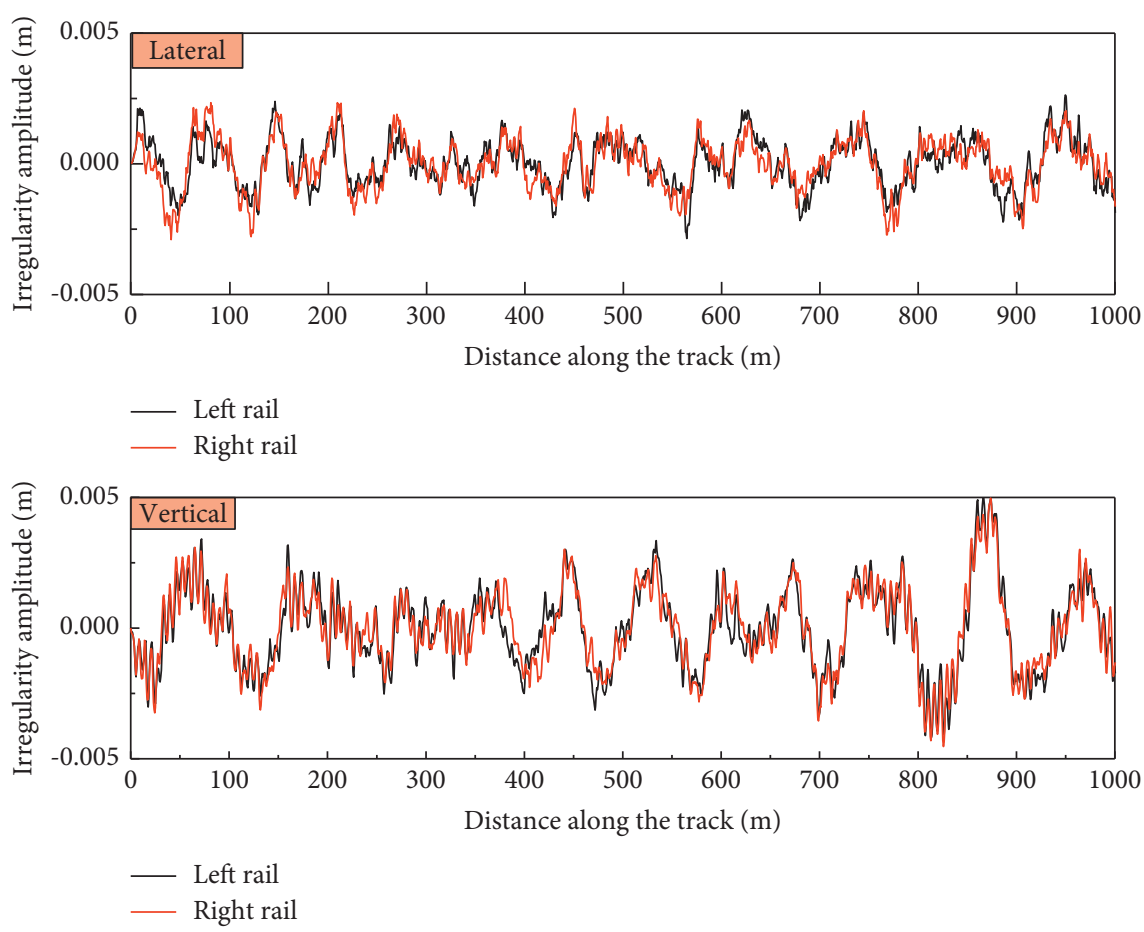

FIgURE 8: The random track irregularity of the Beijing-Tianjin high-speed rail line.

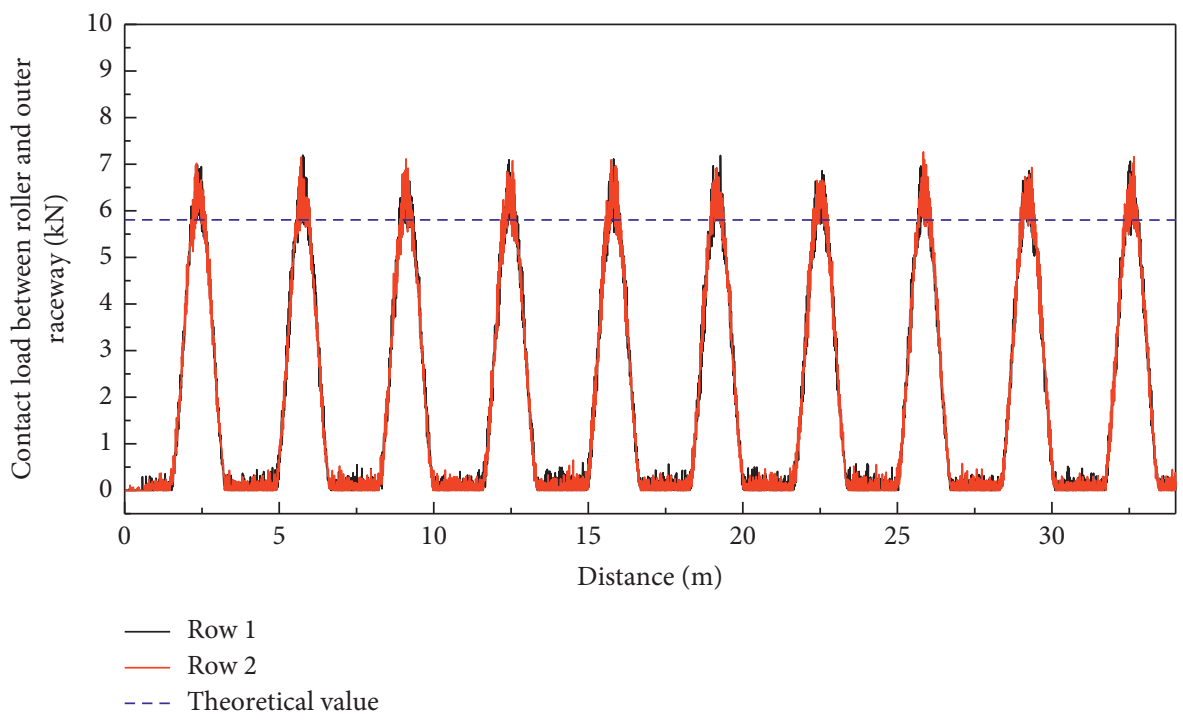

Figure 9: Contact load history of the roller and outer raceway.

4.2. Contact Load Characteristics of the Roller Raceway with Wheel-Polygonal Excitation. In order to investigate the effect of components of an axle-box bearing model on the vehicle system dynamics when the model is excited by wheel-polygonal wear, simulations have been performed at a running speed of $250 \mathrm{~km} / \mathrm{h}$. Once again, the predominant 20th order wheel-polygonal wear pattern is applied, with an amplitude of $0.01 \mathrm{~mm}$ and no phase difference between the two wheels of a wheelset. The contact load history between the rollers of row 1/row 2 and the outer raceway at the same position is obtained, as shown in Figure 12.
The maximum roller-outer raceway contact load is $7.62 \mathrm{kN}$, which appears on row 2 of the tapered roller bearing. Moreover, it is not much different from the maximum contact load without wheel-rail excitation. Therefore, the two rows of double row tapered roller bearings are loaded alternately in the actual operating conditions.

Figure 13 shows the frequency spectrum of the wheelpolygonal excitation load duration. There is little difference in the amplitude of contact load spectrum between no wheel-rail excitation and wheel-polygon excitation, but the 


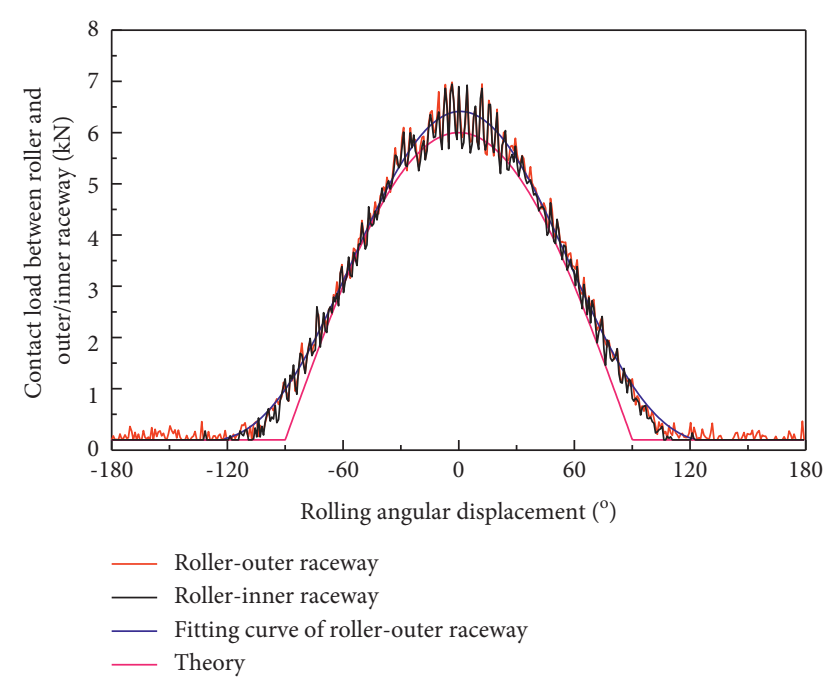

FIGURE 10: Contact load distribution curve between the rollers of row 1 and the inner/outer raceway.

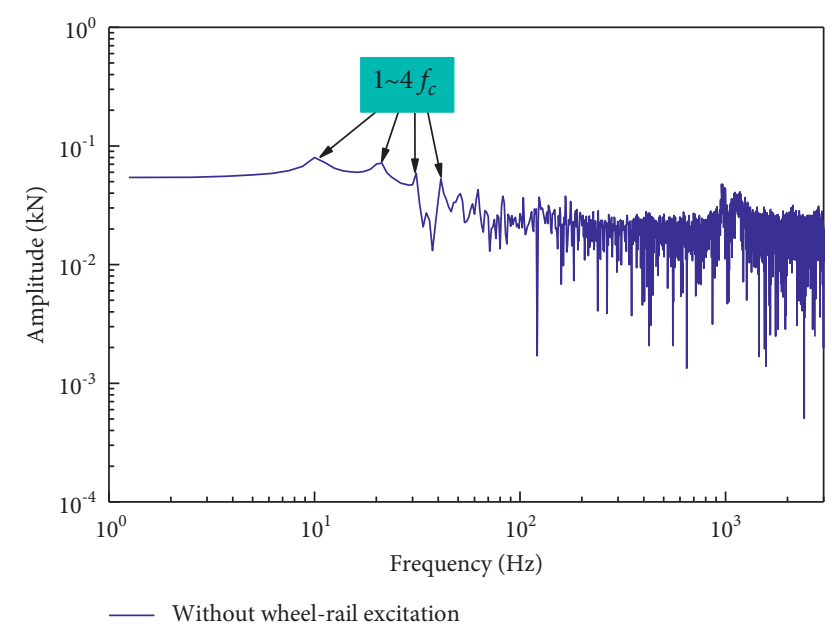

FIgURE 11: Contact load spectrum.

difference, the main frequency of $480 \mathrm{~Hz}$, and its sideband with cage rotation frequency $f_{c}$ can be clearly observed in the contact load spectrum under wheel-polygonal excitation, and this is consistent with the fault characteristic frequency $f_{\mathrm{wd}}$ of 20 order wheel polygon at $250 \mathrm{~km} / \mathrm{h}$.

The fault characteristic frequency of $\mathrm{N}$-order polygon of wheelset can be expressed as

$$
f_{w d}=\frac{v N}{2 \pi R} .
$$

4.3. Contact Load Characteristics of the Roller Raceway with Track Irregularity Excitation. In order to investigate the effect of components of an axle-box bearing model on the vehicle system dynamics when the model is excited by track irregularity excitation, the track irregularity excitation of the Beijing-Tianjin high-speed rail line is added to the simulation calculation. The contact load history between the rollers of row 1/row 2 and the outer raceway at the same position is obtained, as shown in Figure 14.

The two rows of double row tapered roller bearings are loaded alternately, which is due to the axial movement between the inner and outer raceway. The maximum contact load between roller and raceway caused by track irregularity excitation is $8.76 \mathrm{kN}$, which is increased by $20.67 \%$ compared with the contact load without wheel rail excitation.

Figure 15 shows the contact load spectrum under the excitation condition of track irregularity. The frequency component amplitude of the frequency band below about $800 \mathrm{~Hz}$ in the spectrum of load history increases significantly, which indicates that the track irregularity excitation mainly leads to the increase of medium and low frequency contact collision between rollers and raceway.

Moment A and moment B in Figure 14 calculate the instantaneous contact load between the roller and the outer raceway under track irregularity excitation, as shown in Figures 16 and 17.

At moment $\mathrm{A}$, due to the influence of the centrifugal force, half of the rollers of the axle-box bearing are loaded during the operation, and the other half of the rollers are basically not loaded. The maximum contact force is $7.58 \mathrm{kN}$, which appears at the No. 0 roller of the row 2. At moment B, the rollers originally in the loaded zone enter the unloaded zone, and the rollers in the unloaded zone enter the loaded zone. The maximum contact force is $6.38 \mathrm{kN}$, which appears at the No. 8 roller of row 2 .

4.4. Contact Load Characteristics of the Roller Raceway with Comprehensive Excitation. In order to investigate the effect of components of an axle-box bearing model on the vehicle system dynamics when the model is excited by both wheelpolygonal excitation and track irregularity excitation, the roller-outer raceway contact load time history under different working conditions is shown in Figure 18.

It can be seen that the peak values of roller-outer raceway contact force under track irregularity and comprehensive excitation conditions are far greater than those in another two working conditions. Compared with track irregularity excitation, the peak value of roller-outer raceway contact force under comprehensive excitation is increased by approximately $9.77 \%$. Compared with wheel-polygonal excitation, the peak value of roller-outer raceway contact force under comprehensive excitation is increased by approximately $31.25 \%$. Compared with no wheel-rail excitation, the peak value of roller-outer raceway contact force under comprehensive excitation is increased by approximately $38.59 \%$. Hence, the roller's contact force is directly affected by track irregularity excitation.

Figure 19 shows the contact load spectrum under different working conditions. Except for the frequency band near the 20th order wheel-polygon fault frequency, the amplitude of the frequency spectrum has little difference between each frequency band under the conditions of comprehensive excitation and track irregularity excitation. Because the comprehensive excitation includes the wheelpolygon excitation, the sidebands formed by wheel-polygon 


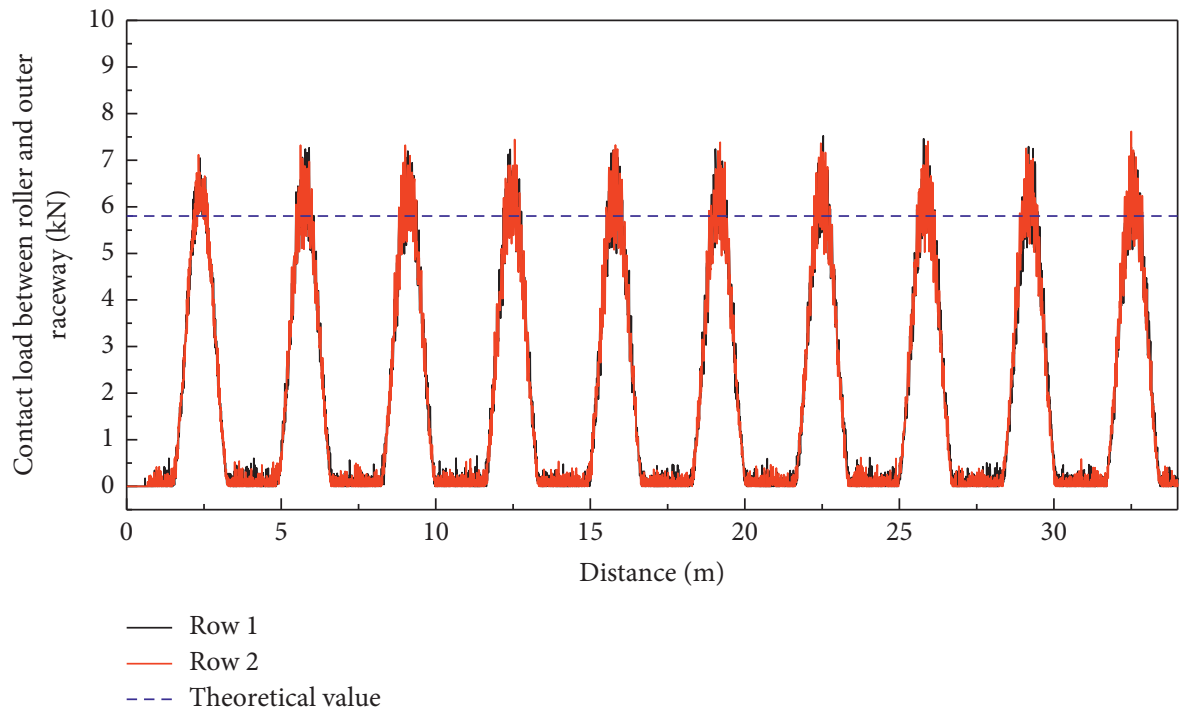

Figure 12: Contact load history of the roller and outer raceway.

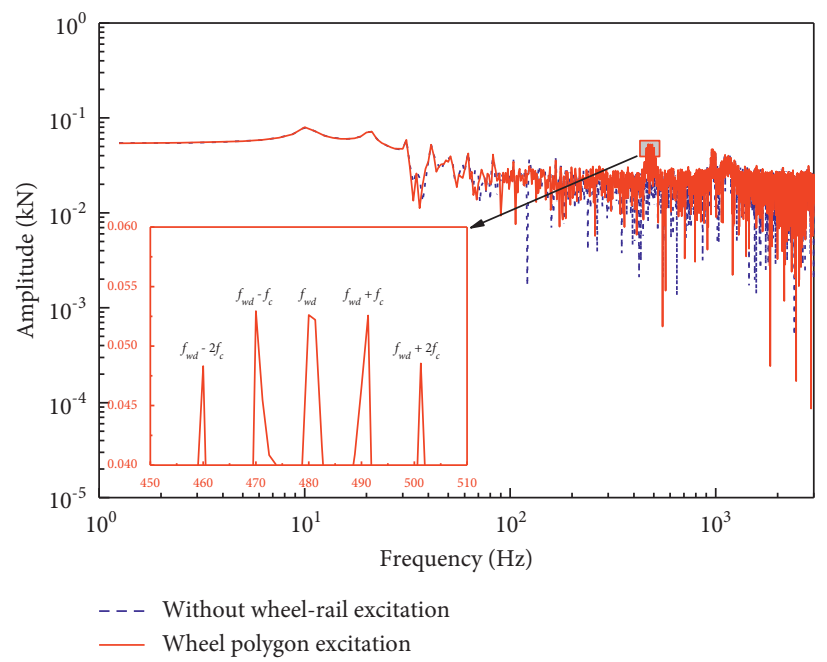

Figure 13: Contact load spectrum.

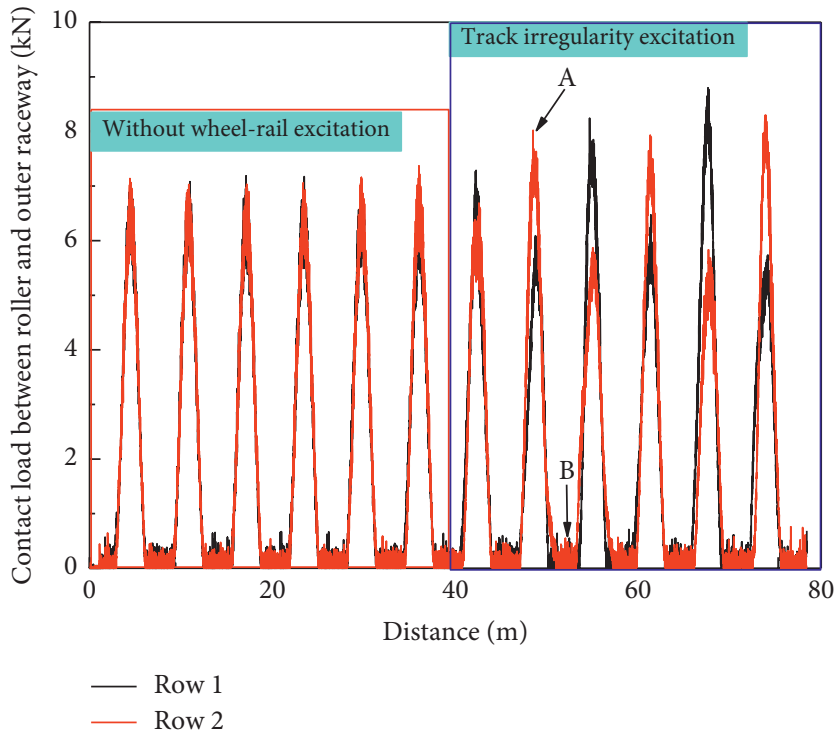

FIgURE 14: Contact load history of the roller and outer raceway. 


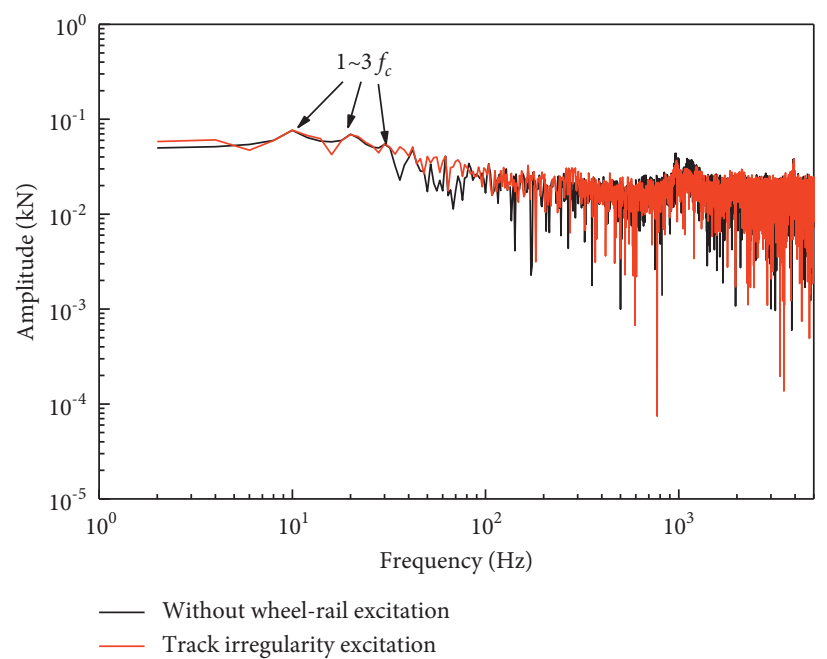

Figure 15: Contact load spectrum.

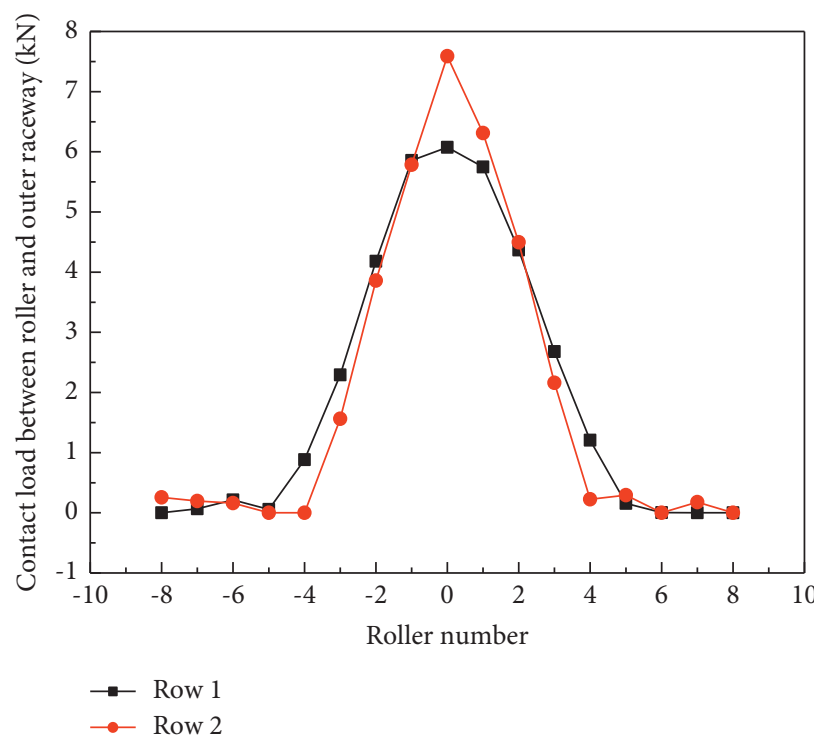

FIGURE 16: Instantaneous contact load (moment A).

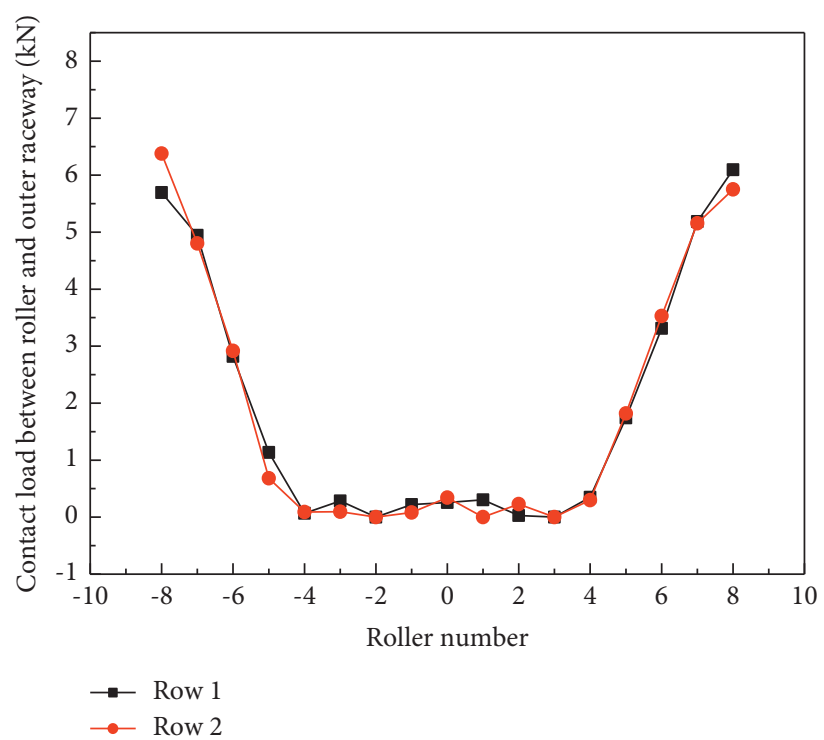

FIGURE 17: Instantaneous contact load (moment B). 


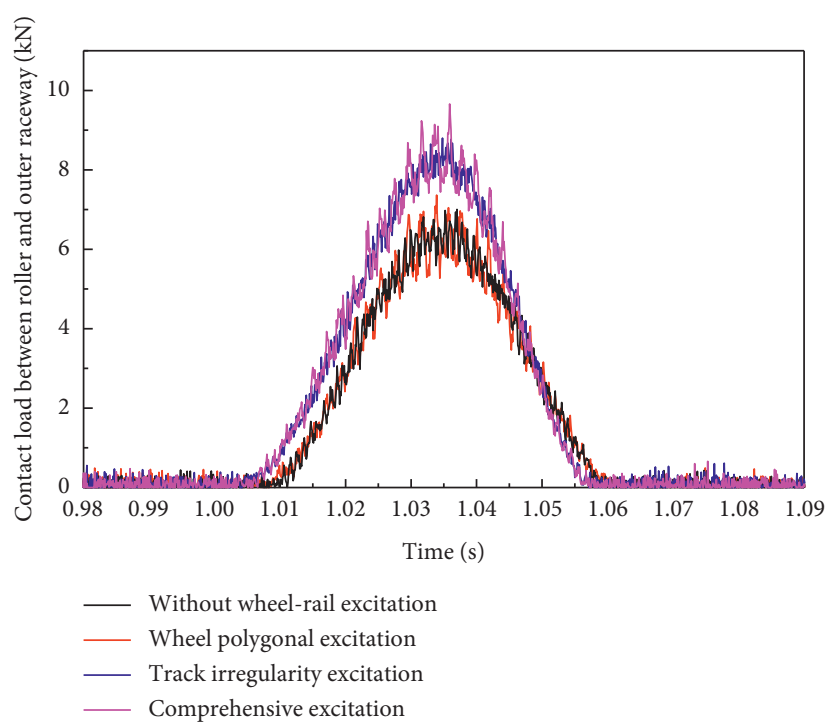

Figure 18: Contact load time history of the roller and outer raceway.

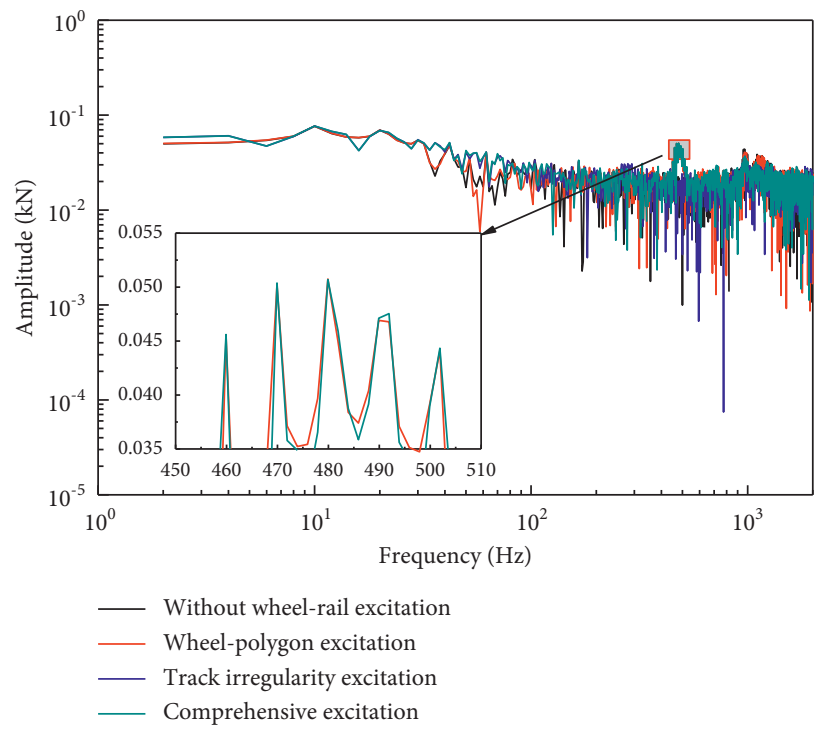

Figure 19: Contact load spectrum.

fault frequency and cage rotation frequency can be obviously observed in the contact load spectrum under the conditions of comprehensive excitation and wheel-polygon excitation.

Under the wheel-rail excitation condition, the contact load of a single roller cannot reflect the contact load characteristics of all rollers. But we can use the statistical method to study the contact load characteristics of the roller through each numbered position of the outer raceway.
Figure 20 presents the max values of the roller-outer raceway contact force, which are obtained under four working conditions at a speed of $250 \mathrm{~km} / \mathrm{h}$.

As can be seen from Figure 20, most of the roller-outer raceway contact load peaks under track irregularity and comprehensive excitation are greater than those under the other two conditions, indicating that the impact of track irregularity on contact load is dominant. 

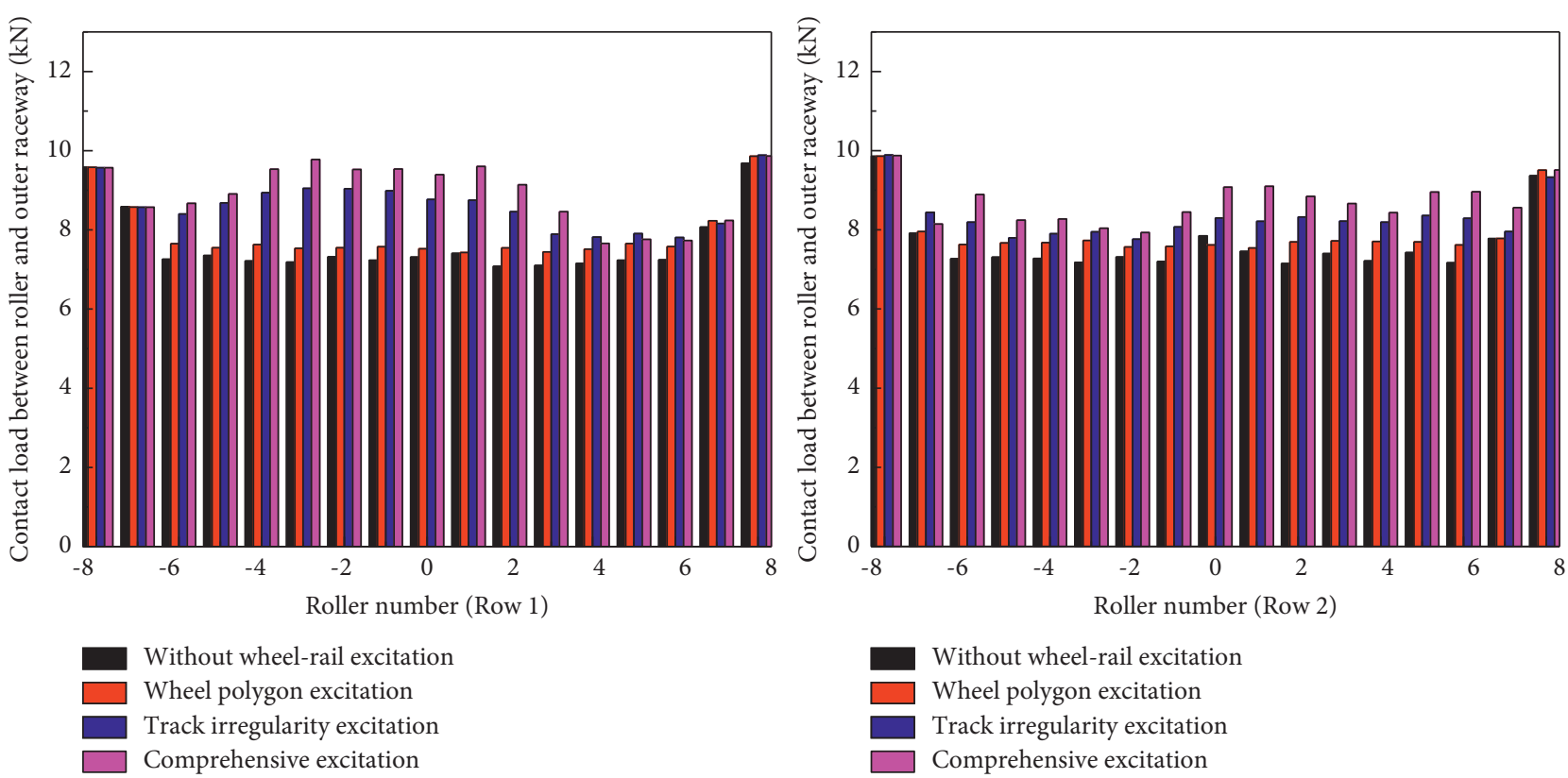

Figure 20: Maximum amplitude of roller-outer raceway contact force.

\section{Conclusions}

To investigate the effects of wheel-rail contact relationship on the dynamic performance of bearing, a vehicle-track coupling dynamic model considering axle-box bearing is built, and the contact relationship between roller and raceway is described in detail. Moreover, field tests are employed to validate the constructed model and good agreement has been demonstrated. Based on the model, the dynamic performance of the axle-box bearing under different working conditions is discussed in this paper. Some conclusions can be drawn:

(1) The two rows of double row tapered roller bearings are loaded alternately in the actual operating conditions.

(2) The track irregularity excitation mainly leads to the increase of medium and low frequency contact collision between rollers and raceway.

(3) Compared with no wheel-rail excitation, the peak value of roller-outer raceway contact force under comprehensive excitation is increased by approximately $38.59 \%$.

(4) Most of the peak values of roller-outer raceway contact force under track irregularity and comprehensive excitation conditions are far greater than those under wheel-polygonal excitation and no wheel-rail excitation, which indicates that the impact of track irregularity on the contact load is dominant

\section{Data Availability}

The experimental data are based on the CRRC project and are confidential.

\section{Conflicts of Interest}

The authors declare no conflicts of interest.

\section{Acknowledgments}

This work was supported by the National Natural Science Foundation of China (no. 51905453), and authors would like to thank the State Key Laboratory of Traction Power for providing office, equipment, and materials to this project.

\section{References}

[1] X. Jiang, X. Cheng, J. Shi, W. Huang, C. Shen, and Z. Zhu, “A new 10-norm embedded MED method for roller element bearing fault diagnosis at early stage of damage," Measurement, vol. 127, pp. 414-424, 2018.

[2] A. Palmgren, Ball and Roller Bearing Engineering, SKF Industries Inc, Philadelphia, 1959.

[3] A. B. Jones, "A general theory for elastically constrained ball and radial roller bearings under arbitrary load and speed conditions," J Basic Eng, vol. 82, no. 21, pp. 309-320, 1960.

[4] T. A. Harris, Rolling Bearing Analysis, John Wiley \& Sons, New York, 2nd ed. edition, 1984.

[5] S. Andréason, "Load distribution in a taper roller bearing arrangement considering misalignment," Tribology, vol. 6, no. 3, pp. 84-92, 1973.

[6] J. Y. Liu, "Analysis of tapered roller bearings considering high speed and combined loading," Journal of Lubrication Technology, vol. 98, no. 4, pp. 564-572, 1976.

[7] V.-C. Tong and S.-W. Hong, "Characteristics of tapered roller bearing subjected to combined radial and moment loads," International journal of Precision Engineering and Manufacturing-Green Technology, vol. 1, no. 4, pp. 323-328, 2014.

[8] L. Houpert, "An enhanced study of the load-displacement relationships for rolling element bearings," Journal of Tribology, vol. 136, no. 1, pp. 01-11, 2014.

[9] V. K. Garg and R. V. Dukkipati, Dynamics of Railway Vehicle Systems, Academic Press, Toronto, 1984.

[10] A. H. Wickens, Fundamentals of Rail Vehicle Dynamics: Guidance and Stability, Swets \&Zeitlinger, Lisse, 2003. 
[11] W. M. Zhai and X. Sun, "A detailed model for investigating vertical interaction between railway vehicle and track," Vehicle System Dynamics, vol. 23, no. 1, pp. 603-615, 1994.

[12] W. Zhai, K. Wang, and C. Cai, "Fundamentals of vehicle-track coupled dynamics," Vehicle System Dynamics, vol. 47, no. 11, pp. 1349-1376, 2009.

[13] G. Diana, F. Cheli, S. Bruni, and A. Collina, "Interaction between railroad superstructure and railway vehicles," Vehicle System Dynamics, vol. 23, no. 1, pp. 75-86, 1994.

[14] J. C. O. Nielsen and A. Igeland, "Vertical dynamic interaction between train and track influence of wheel and track imperfections," Journal of Sound and Vibration, vol. 187, no. 5, pp. 825-839, 1995.

[15] N. Chaar and M. Berg, "Simulation of vehicle-track interaction with flexible wheelsets, moving track models and field tests," Vehicle System Dynamics, vol. 44, no. 1, pp. 921-931, 2007.

[16] Z. Wang, G. Mei, Q. Xiong, Z. Yin, and W. Zhang, "Motor car-track spatial coupled dynamics model of a high-speed train with traction transmission systems," Mechanism and Machine Theory, vol. 137, pp. 386-403, 2019.

[17] Z. Wang, W. Zhang, Z. Yin, Y. Cheng, G. Huang, and H. Zou, "Effect of vehicle vibration environment of high-speed train on dynamic performance of axle box bearing," Vehicle System Dynamics, vol. 57, no. 4, pp. 543-563, 2019.

[18] H. Zha, Z. S. Ren, and R. Xue, "Study on cumulative damage and fatigue life of axle box bearings in high-speed EMU," Journal of the China Railway Society, vol. 40, no. 10, pp. 30-35, 2018.

[19] H. Zha, Z. S. Ren, and N. Xu, "Load characteristics of axle box bearing raceway of high-speed EMU," Journal of Mechanical Engineering, vol. 56, no. 4, pp. 135-142, 2020.

[20] Z. Wang, P. Allen, G. Mei, R. Wang, Z. Yin, and W. Zhang, "Influence of wheel-polygonal wear on the dynamic forces within the axle-box bearing of a high-speed train," Vehicle System Dynamics, vol. 58, no. 9, pp. 1385-1406, 2020.

[21] X. Jiang, S. Li, and C. Cheng, "A novel method for adaptive multiresonance bands detection based on VMD and using MTEO to enhance rolling element bearing fault diagnosis," Shock and Vibration, vol. 2016, pp. 1-20, 2016.

[22] W. H. Zhang, Dynamics of Coupled Systems in High-Speed Trains: Theory and Practice, Science press, Beijing, 2013. 